\title{
DIFFRACTION BASED MEASUREMENT OF SURFACE MACHINING DAMAGE IN SUPERALLOYS
}

\author{
M.A. Othon, L.N. Brewer, and Y. Gao \\ GE Global Research Center, Niskayuna, NY 12309
}

\section{B.T. Hazel, W.H. Buttrill \\ GE Aircraft Engines, Cincinnati, OH 45215}

Most machinery components require some amount of machining during their construction. In a general sense, machining involves cutting, grinding, and polishing the surface of the metal component. The mechanical properties and service life of the component can be greatly affected by residual surface damage from the machining process. It is difficult to know how much residual damage is left in the surface of the metal as a result of these processes. In this paper, we have applied electron backscattered diffraction (EBSD) and synchrotron X-ray diffraction to quantify the level of residual damage (plastic strain) as a function of distance from the surface of the component.

The measurements were made on components fashioned from a Fe-Ni-based superalloy. The components were machined using a set of increasingly aggressive parameters to intentionally increase the amount of damage on the surface of the material. Cross-sectional samples were cut from these components and polished down to a $0.05 \mu \mathrm{m}$ silica finish on the cross-sectional face. EBSD measurements were performed at the GE Global Research Center using a CamScan CS44 SEM with an HKL-Channel 5 EBSD system. EBSD data was collected from the samples in lines parallel to the surface of the component (Figure 1). The average intragrain misorientation (AMIS) was calculated for each line and plotted as a function of distance from the surface as has been done previously for studies on welds [1] (Figure 2). X-ray diffraction (XRD) measurements were made using high-energy $(50 \mathrm{keV})$ synchrotron X-rays at the National Synchrotron Light Source. The diffraction was performed in transmission using the same samples as the EBSD measurements but sliced to be $\sim 1 \mathrm{~mm}$ thick (Figure 3) [2]. The sample was translated through the beam $(0.015 \mathrm{x} 2 \mathrm{~mm}$ in cross-section) perpendicular to the surface to achieve a sampling of points as a function of depth from the surface. A CCD camera was used to record the diffraction rings from each point on the sample. From this data, the width of the diffraction lines (sensitive to plastic strain (Figure 4)) and the positions of the diffraction lines (sensitive to elastic strain) were measured.

Both EBSD and XRD were able to measure distinct distributions of plastic strain for the medium and highly aggressive conditions. In both EBSD and X-ray diffraction data, the plastic strain is observed to decay from significant levels at the surface to baseline levels about 300-400um from the surface for the most aggressive machining conditions. For the least aggressive conditions, no change in EBSD-AMIS value was observed as a function of depth. XRD measurements also suggest that these plastic strain distributions give rise to significant levels of elastic residual stress that depend upon the surface machining procedures.

Acknowledgement: YG wishes to thank Dr. Z. Zhong of National Synchrotron Light Source for his assistance in performing the synchrotron measurement.

[1] L.M. Young, P.L. Andresen and T.M. Angeliu, Corrosion/01, paper 131, NACE, Houston, TX , 2001.

[2] W. Reimers, et. al., J. Materials Science Lett. 18 (1999) 5821-583. 
Figure 1.

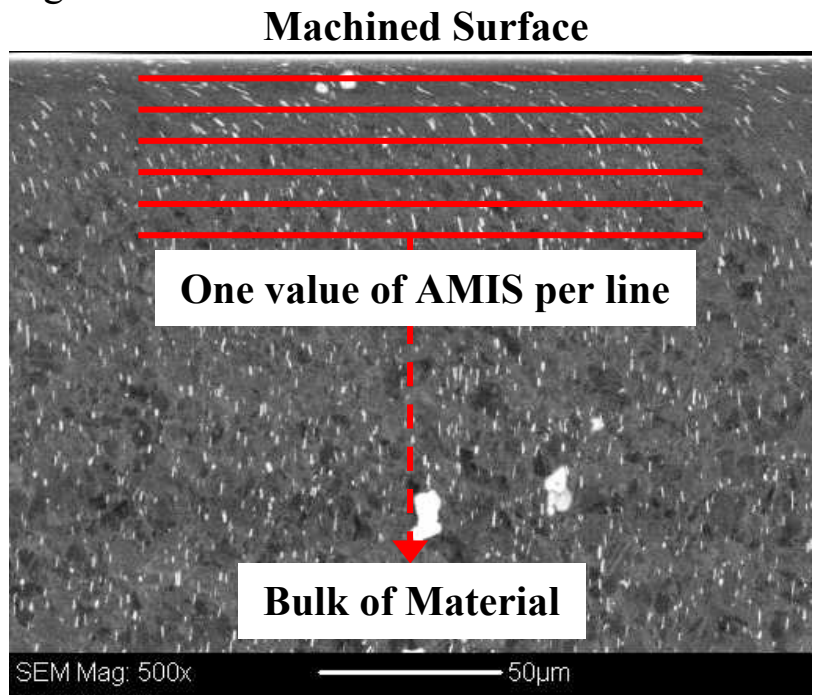

Figure 2.

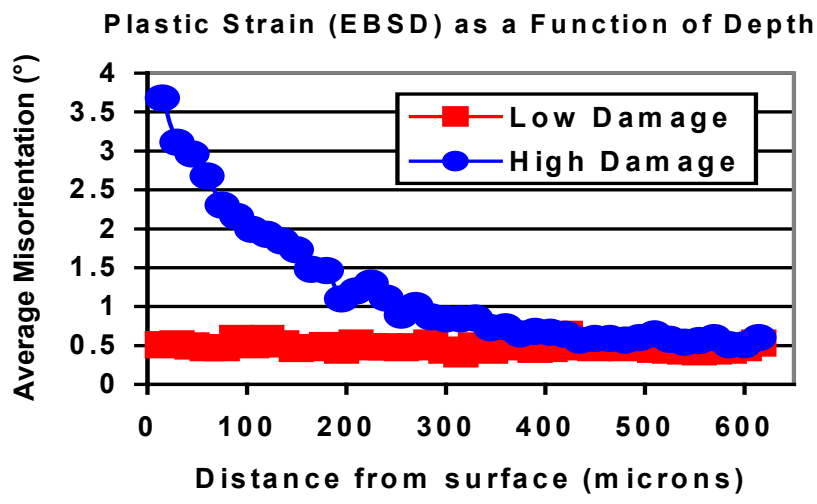

Figure 4.

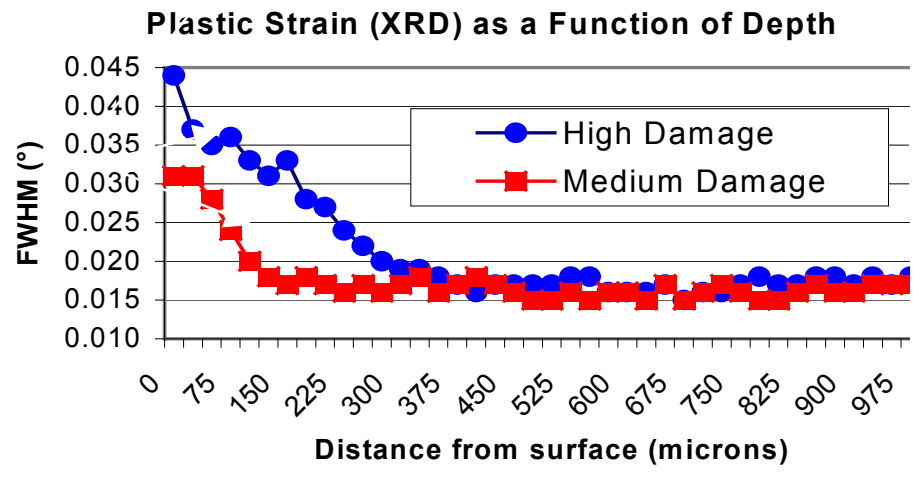

Figure 1. Schematic for EBSD measurement of surface damage. Each line is $150 \mu \mathrm{m}$ in length. Separation between lines is $15 \mu \mathrm{m}$.

Figure 2. Plastic strain using EBSD-AMIS as a function of depth from machined surface for different machining conditions

Figure 3. Experimental geometry for synchrotron X-ray diffraction experiments.

Figure 4. Plastic strain using X-ray diffraction peak width as a function of depth from machined surface for different machining conditions. 